\title{
La participación social en la reforma educativa en México 2012-2018
}

Social participation in educational reform in Mexico 2012-2018

\author{
Volumen 20, Número 3 \\ Setiembre - Diciembre \\ pp. 1-28
}

\section{Ursula Zurita Rivera}

Citar este documento según modelo APA

Zurita Rivera, Ursula. (2020). La participación social en la reforma educativa en México 20122018. Revista Actualidades Investigativas en Educación, 20(3), 1-28. Doi. 10.15517/aie.v20i3.43673 


\title{
La participación social en la reforma educativa en México 2012-2018
} Social participation in educational reform in Mexico 2012-2018

\section{Ursula Zurita Rivera'}

\begin{abstract}
Resumen: En este ensayo se examina cómo la participación en las escuelas fue formulada en la reforma educativa emprendida en México en 2012-2018. La intención es conocer algunas de las principales implicaciones derivadas de su concepción como un medio para fortalecer la educación pública, laica y gratuita; asegurar una mayor equidad en el acceso a una educación de calidad; fortalecer la capacidad de gestión de las escuelas; instaurar la evaluación de los distintos componentes del sistema educativo y establecer el servicio profesional docente, los cuales confluirían en la implementación del Proyecto de la Escuela al Centro y del Nuevo Modelo Educativo. El presupuesto central es que la visión instrumental y pragmática de la participación se materializó en las escuelas a través de la operación del Programa de la Reforma Educativa, el de Escuelas de Tiempo Completo y el de Convivencia Escolar, develando rápidamente las contradicciones y limitaciones de la reforma en esta materia. El ensayo expresa que esa concepción preservó y fortaleció la preminencia del Estado mexicano en los procesos participativos, la producción y reproducción de una seudo autonomía social, como también, de una participación subordinada, simulada y pasiva, anclada en una lógica vertical, burocrática y jerárquica del sistema educativo mexicano. En menoscabo de expresiones autónomas e independientes de actores, como padres de familia y alumnado que históricamente han sido supeditados e invisibilizados a pesar de su potencial contribución a la ampliación y profundización del derecho a la educación, la educación para la vida democrática y la inclusión educativa.
\end{abstract}

Palabras clave: participación social, reforma educativa, México

\begin{abstract}
This essay examines how participation in schools was formulated in the educational reform undertaken in Mexico in 2012-2018. The intention is to know some of the main implications derived from its conception as a means to strengthen public, secular and free education, to ensure greater equity in access to quality education, to strengthen the management capacity of schools, establish the evaluation of the different components of the educational system and establish the professional teaching service that would converge in the implementation of the Project from the School to the Center and the New Educational Model. The central assumption is that the instrumental and pragmatic vision of participation was materialized in the schools through the operation of the Educational Reform Program, the Full-Time Schools Program, and the School Coexistence Program, quickly revealing the contradictions and limitations of the reform in this matter. The essay expresses that this conception preserved and strengthened the preeminence of the Mexican State in participatory processes, the production, and reproduction of a pseudo-social autonomy, as well as a subordinate, simulated, and passive participation, anchored in a vertical, bureaucratic and hierarchical logic of the Mexican educational system. Undermining the autonomous and independent expressions of actors such as parents and students who have historically been subordinated and made invisible despite their potential contribution to the expansion and deepening of the right to education, education for democratic life, and educational inclusion.
\end{abstract}

Key words: social participation, education reform, Mexico

1 Profesora investigadora de tiempo completo de la Facultad
Latinoamericana de Ciencias Sociales Sede Académica México, Ciudad
de México, México. Doctora en Ciencia Política en la Universidad
Nacional Autónoma de México, México. Orcid: https://orcid.org/0000-
0002-7381-3550

Dirección electrónica: uzurita@flacso.edu.mx

Ensayo recibido: 11 de mayo, 2020

Enviado a corrección: 6 de julio, 2020

Aprobado: 26 de agosto, 2020 


\section{Introducción}

La participación social en la educación y en las escuelas ha estado presente desde los ideales sociales y políticos de la Revolución Mexicana en 1910 hasta un siglo después en el marco de la reforma educativa instaurada entre 2012 y 2018. Durante más de un siglo se puede observar cómo se han desplegado diversas concepciones y usos de la noción participación social en la construcción y consolidación del sistema educativo, así como en acompañar y reforzar procesos políticos, sociales, económicos y culturales que se pretendía alcanzar a través de proyectos de gran relevancia que trascendían el sector educativo, pero que tenían fuertes efectos en él. Así, alrededor de la identidad nacional, la modernización, la reforma del estado, la democratización o la crisis fiscal, entre otros acontecimientos nacionales se fueron trazando disímiles sentidos acerca del tipo de participación social que se buscaba, y las razones que las autoridades educativas aducían al respecto (Barba, 1998; 2007; Latapí, 1998; 2005; Zorrilla y Barba, 2008; Zurita, 2015; 2018c).

Si bien, al hacer un recuento de esos múltiples sentidos se puede advertir que, en algunos momentos históricos, hubo visiones más precisas y claras acerca de lo que se entendía y se pretendía, como la descentralización educativa a principios de los años noventa, también hubo otros momentos donde las administraciones federales tuvieron ideas un tanto confusas e, incluso, contradictorias entre sí (Latapí, 2005). Sin embargo, esta aparente paradoja no es un rasgo exclusivo del Estado mexicano, múltiples especialistas han dicho que la complejidad de la participación social se debe a su inherente carácter polisémico, que la lleva a estar presente en discursos y políticas educativas que apelan a las más distintas y opuestas ideologías (Corvalán y Fernández, 2000; Dagnino, 2006; Font, 2004; Gajardo, 1999; López, 2006; Torres, 2001).

En este sentido, el gobierno federal del 2012 al 2018 tuvo una postura sobre la participación social en la educación y sobre las escuelas que conviene examinar aquí. Tan pronto como asumió el poder en 2012, el entonces presidente Enrique Peña Nieto estableció una alianza entre los tres principales partidos políticos, la cual fue conocida como Pacto por México (Elizondo, 2017). Esta alianza le permitió impulsar un paquete de once reformas estructurales en varios sectores cruciales de la administración pública. Una de las que más avanzó fue, precisamente, la reforma educativa, que adquirió un rango constitucional por los cambios introducidos en la Constitución Política de los Estados Unidos Mexicanos y en la Ley General de Educación, así como en el sustento jurídico que se le dio en las Leyes 
Generales del Servicio Profesional Docente y del Instituto Nacional para la Evaluación de la Educación, además de en numerosos lineamentos normativos del sistema educativo (Martínez y Navarro, 2018). La transformación de las escuelas del tramo obligatorio, que por primera vez abarcó del nivel preescolar hasta la educación media superior, estuvo anclada en dos de los propósitos que generaron mayor polémica por el carácter trascendental que tenía en la historia del sistema educativo mexicano, a saber: la instauración de la evaluación educativa para el ingreso, permanencia y ascenso de docentes y el establecimiento del servicio profesional docente (Martínez y Navarro, 2018). Como se puede imaginar, las nuevas reglas institucionales de la evaluación docente implicaron cambios en la organización y gestión del sistema educativo. Fue ahí donde se alojó la propuesta de la participación social para apoyar los otros objetivos de la reforma, como el fortalecimiento de la educación pública, laica y gratuita; el aseguramiento de mayor equidad en el acceso a una educación de calidad, y el impulso de la capacidad de gestión de las escuelas que, junto con la instauración de la evaluación de los distintos componentes del sistema educativo y el establecimiento del servicio profesional docente, confluirían en la implementación del Proyecto de la Escuela al Centro y del Nuevo Modelo Educativo para la Educación Obligatoria.

A partir de esto, se observa que la participación social, junto con la propuesta de autonomía de la gestión escolar, fueron objeto de una reformulación que fue plasmada de manera congruente con las políticas, los programas y otras acciones que se desplegaron durante los seis años (Zurita, 2013, 2018a, 2018b). La reformulación consistió en asumir la participación social desde una visión instrumentalista, pragmática y subordinada a la instauración de la evaluación educativa y del servicio profesional docente. El impulso de la participación se convirtió en un mecanismo para asegurar, desde el espacio escolar, la recuperación de la rectoría de la educación por parte del Estado mexicano a través del fomento de la participación de ciertos actores y, a la vez, de la limitación del poder y la autoridad de otros. De este modo, la reforma educativa de nueva cuenta reprodujo prácticas históricamente ancladas en el sistema educativo en torno a la participación social: por un lado, se subordinó y supeditó la participación de madres y padres de familia a cuestiones de carácter administrativo y operativo de diversos programas estratégicos; y, por otro lado, se marginaron e, inclusive, se excluyeron niñas, niños y adolescentes (NNyA), a pesar de su potencial contribución a la ampliación y profundización del derecho a la educación, la 
educación para la vida democrática y la inclusión educativa (Organización de las Naciones Unidas para la Educación, la Ciencia y la Cultura [UNESCO], 2017).

En este ensayo se examina cómo la participación social en la educación y en las escuelas fue formulada de modo instrumental y pragmático en el marco de la reforma educativa en México emprendida en el periodo 2012-2018. El objetivo principal es identificar algunas de las principales implicaciones de pensar la participación social como un medio para la consecución de múltiples propósitos de la reforma a través de la implementación del Programa de la Reforma Educativa, el Programa de las Escuelas de Tiempo Completo y el Programa Nacional de Convivencia Escolar. Si bien, los dos primeros programas existían desde administraciones anteriores, rápidamente fueron alineados de manera congruente y consistente con el discurso de la reforma educativa y, por ende, acordes con las decisiones tomadas por las autoridades educativas respecto a los integrantes, las acciones y los tipos de participación social que se deseaba impulsar en las escuelas. Ahora bien, en concordancia con el objetivo de este ensayo, aquí se pone la atención en actores individuales como NNyA, madres y padres de familia; mientras que de las modalidades institucionales de participación social se consideran fundamentalmente los Consejos Escolares de Participación Social porque ellos fueron la referencia más notoria de la reforma educativa.

Para analizar la participación de las NNyA en las escuelas es inevitable acudir a la perspectiva de las Voces del Alumnado (Fielding, 2007, 2011; Lundy, 2007; Mitra y Serriere, 2012; Mockler y Groundwater-Smith, 2015; entre otros) para identificar lo que se ha avanzado en este proceso, pero, esencialmente, para tener en cuenta los enormes retos que todavía perduran, aun cuando la participación de NNyA ha sido un compromiso que el Estado mexicano asumió por su adhesión a la Convención de los Derechos del Niño en 1989 y, más, al ser reconocido el derecho a la participación de estas poblaciones, en el 2014, con la expedición de la Ley General de Derechos de Niñas, Niños y Adolescentes. Las Voces del Alumnado cuestionan las concepciones tradicionales de NNyA como personas pasivas y subordinadas que, por esta condición, se les impide desarrollar sus potencialidades individuales, expresar sus opiniones, aportar sus conocimientos, manifestar sus necesidades, tomar decisiones y ser parte de las acciones. Para especialistas de este enfoque, el fracaso de diversas reformas se debe a que, si bien pretenden transformar los procesos de enseñanza y aprendizaje, suelen ignorar voces, opiniones, expectativas, intereses y valoraciones estudiantiles (MacBeath, 2006; McCluskey, et al., 2013; Mitra y 
Serriere, 2012). De manera puntual, Michael Fielding, destacado representante de las Voces del Alumnado, ha cuestionado las nociones en torno a la participación limitada y periférica de NNyA, que han permanecido en las sociedades desde épocas lejanas. Para él, es urgente desmontar esas ideas para impulsar transformaciones profundas que impliquen avanzar hacia concepciones más activas, propositivas y críticas del alumnado, es decir, visiones acordes con el modelo de democracia participativa. Sin esas concepciones será imposible, según Fielding (2011), que NNyA sean concebidos como verdaderos sujetos sociales con agencia, y como sujetos capaces de producir conocimiento. Por ahora, en torno a la participación de NNyA, se advierte la tensión entre modelos autoritarios y otros democráticos. Entre estos dos polos han surgido combinaciones sui generis, tal como sucede en el ámbito educativo en México, pues, a pesar de contar con una legislación que reconoce la participación de NNyA en las escuelas, se condiciona su ejercicio a ciertos rasgos individuales (edad, madurez, desarrollo cognitivo), y se conserva la prohibición establecida desde 1942, en la Ley Orgánica de la Educación Pública, a intervenir en la dirección y el gobierno de las escuelas (Gobierno de los Estados Unidos Mexicanos, 1942).

Por otro lado, es preciso decir que, a pesar de la diversidad de los contextos geográficos y los desafíos educativos que se enfrentan en cada sociedad, desde las últimas décadas hay un gran consenso acerca del profundo impacto que tiene la participación de madres y padres de familia en el aprendizaje; la permanencia; la conclusión de los estudios; la satisfacción y cumplimiento de las expectativas educativas; la prevención de la violencia escolar; la autonomía y gestión escolar; la descentralización; el desempeño docente; el liderazgo directivo; el clima escolar; entre otros aspectos (Hornby y Lafaele, 2011). Con la intención de conocer ese impacto, se han emprendido estudios empíricos basados en la aplicación de distintas técnicas investigativas en una gama heterogénea de poblaciones, escuelas, sistemas educativos, contextos geográficos y periodos históricos. Además, se han desarrollado varios modelos teóricos para examinar diferentes tipos de participación de padres y madres de familia (Epstein, Clark, Salinas y Sanders, 1997), así como para identificar los elementos clave que fomentan u obstaculizan dicha participación en el contexto escolar (Hornby y Lafaele, 2011). En esta discusión no están ausentes los cambios sociales que recientemente han afectado los principios, valores, expectativas y sentidos de la participación de madres y padres de familia, en torno a la educación, suscitados por las transformaciones en la composición, estructura y funcionamiento de las familias contemporáneas (Doepke y Zilibotti, 2019). Ante tal complejidad, los modelos que tienen 
pretensiones explicativas suelen retomar diferentes dimensiones individuales, escolares, educativas y sociales que inciden en la configuración de la participación de padres y madres de familia. Con ello, se trascienden las visiones simplistas que asumían que la participación, fundamentalmente, es producto de la voluntad de los actores o de la primacía de un factor individual para determinar la participación de las familias (Hornby y Lafaele, 2011). Así, las investigaciones contemporáneas han intentado profundizar en las formas en que esas dimensiones se interrelacionan en el despliegue de experiencias participativas donde madres y padres de familia tienen un lugar primordial. Con todo, la experiencia de México muestra cómo el propio sistema educativo ha diseñado mecanismos institucionales que obstaculizan y limitan la participación de madres y padres. Aunque estos integrantes de las comunidades escolares cuentan con las Asociaciones de Padres de Familia como la vía primordial para participar en el ámbito escolar en México, su diseño ha permanecido intacto desde los años ochenta en que fueron formuladas. De modo tal que no se han incorporado los cambios experimentados por las familias mexicanas, y siguen siendo concebidas como entes para la recaudación de cuotas, reparar el inmobiliario escolar, suministrar recursos materiales. Aunado a ello, en México la participación de las familias ha sido históricamente utilizada para vigilar, fiscalizar y limitar el poder de la autoridad escolar (Barba, 1998; 2007; Latapí, 1998; 2005; Zorrilla y Barba, 2008; Zurita, 2015; 2018c).

Ahora bien, para realizar este ensayo se revisaron diferentes documentos relacionados con la implementación de programas estratégicos del gobierno federal, dado que el presupuesto central es que la visión instrumental y pragmática de la participación se materializó en las escuelas a través de la operación del Programa de la Reforma Educativa, el Programa Escuelas de Tiempo Completo y el Programa Nacional de Convivencia Escolar, que no tardaron en develar las contradicciones, limitaciones y tensiones de la reforma en esta materia. En especial, se pone la atención en la participación individual y/o colectiva que habría de tener un protagonismo destacado en la implementación de tales iniciativas, ya sea por su presencia protagónica o por su ausencia misma. A partir de ello, y tomando en cuenta la literatura especializada, se procederá a discutir los sentidos y los desafíos principales de la participación social desplegados por la reforma educativa que Enrique Peña Nieto impulsó. Por último, se expondrán algunas reflexiones con la intención de contribuir a la discusión sobre los alcances que se desprenden de esta singular experiencia que vivieron las escuelas públicas mexicanas del tramo obligatorio en los últimos años. 


\section{La participación social en la reforma educativa 2012-2018}

En la reforma educativa en México, lanzada en el periodo 2012-2018, la participación social tuvo un lugar importante, pero desde el inicio se advirtió que este papel estaría supeditado a dos de sus tópicos más polémicos, a saber: la evaluación educativa y el servicio profesional docente (Martínez y Navarro, 2018). En materia de participación social, los esfuerzos para replantear sus significados con la reforma educativa, básicamente, se concentraron en la figura de los Consejos de Participación Social y su articulación con la gestión escolar, a partir de los cuales se estipularon los fundamentos para la autonomía de la gestión escolar en donde fue fincado el Proyecto de la Escuela al Centro. En esta reformulación normativa se incluyeron modificaciones estrechamente vinculadas con los propósitos de la reforma educativa del 2012-2018. En otras palabras, dentro de dicho Proyecto, la participación social fue articulada con los programas de gestión, con el fin de proporcionar los apoyos necesarios para que las escuelas aseguraran una normalidad mínima de operación; contaran con las plantillas de personal completas al inicio de cada ciclo escolar; garantizaran el aprovechamiento óptimo del tiempo escolar; aligeraran la carga administrativa a las escuelas; e impidieran el involucramiento de las escuelas en cualquier tipo de iniciativas públicas, sociales o privadas que las desviaran del cumplimiento de sus fines educativos; entre otros propósitos.

Es por ello que dentro de las primeras acciones emprendidas se halla la firma del Acuerdo Secretarial 716 que establece lineamientos para la constitución, organización y funcionamiento de los Consejos de Participación Social en la Educación el 4 de marzo de 2014 (Gobierno de los Estados Unidos Mexicanos, Secretaría de Educación Pública, 2014a) y la del Acuerdo Secretarial 717 por el que se establecen los lineamientos para formular los Programas de Gestión Escolar (Gobierno de los Estados Unidos Mexicanos, Secretaría de Educación Pública, 2014b). En los años siguientes, el Acuerdo Secretarial 716, fue objeto de algunos cambios que dieron lugar al Acuerdo 02/05/2016 (Gobierno de los Estados Unidos Mexicanos, Secretaría de Educación Pública, 2016), y meses después, al Acuerdo 08/08/2017 (Gobierno de los Estados Unidos Mexicanos, Secretaría de Educación Pública, 2017a). Con la primera intención de emprender acciones para promover la simplificación y la actuación de los Consejos (Acuerdo Secretarial 716, artículo $1^{\circ}$ ), estos fueron definidos en el artículo $2^{\circ}$ del Acuerdo Secretarial 716 como "instancias de participación social en la 
educación, de consulta, orientación, colaboración, apoyo e información, según corresponda, con el propósito de participar en actividades tendientes a fortalecer, ampliar la cobertura y elevar la calidad y la equidad en la educación básica" (Gobierno de los Estados Unidos Mexicanos, Secretaría de Educación Pública, 2014a, p. 1). En el Acuerdo 716 y Acuerdos posteriores, se dio respuesta a cuestionamientos y críticas en torno a los Consejos en administraciones federales previas relativas a: 1) simplificar la actuación de los Consejos Escolares, Municipales, Estatales y Nacional de Participación Social; 2) reconocer una opción diferente para la conformación del Consejo en escuelas de integración incompleta, unitarias o bidocentes; 3) disminuir el número de los consejeros; 4) modificar la designación, renovación, duración y remoción según el tipo de representantes en cada Consejo; 5) difundir los proyectos elaborados por los diferentes Consejos; y, 6) promover la vinculación entre los Consejos y la sociedad mexicana (Zurita, 2015). Sin embargo, continuaron indefinidos varios aspectos cruciales que obstaculizaron su existencia en los términos deseados, como aquellos relacionados con fuentes, montos y disponibilidad de recursos financieros, materiales y humanos necesarios para su operación.

El análisis del contenido de los Acuerdos Secretariales 716, 02/05/2016 y 08/08/2017 (Gobierno de los Estados Unidos Mexicanos, Secretaría de Educación Pública, 2014a; 2016, 2017a) muestra que la participación social impulsada por la reforma estuvo localizada circunscrita fundamentalmente a la dimensión escolar. Esta delimitación, cabe agregar, fue consistente tanto con el alcance de los cambios normativos sobre el funcionamiento de los Consejos Escolares de Participación Social, como con el fomento de un modelo de autonomía de la gestión y de una autonomía curricular en las instituciones escolares (Zurita, 2018c). Así, dentro de la gama de instrumentos del marco normativo que dieron forma al Proyecto de la Escuela al Centro y del Nuevo Modelo Educativo, se confirma el carácter protagónico que tendría que encarnar la persona responsable de la dirección escolar en las decisiones y acciones vinculadas con el empleo de recursos, la planeación, la participación social y el fortalecimiento de la autonomía de la gestión escolar. A esa persona, además, se le asignó la responsabilidad, junto con el Consejo Técnico Escolar, la Asociación de Padres de Familia, el Consejo Escolar de Participación Social o las Asociaciones Promotoras de Educación Comunitaria, de transparentar y rendir cuentas a la comunidad y a las autoridades educativas respectivas de los procesos y recursos, los aspectos educativos y administrativos que en su conjunto habrían de robustecer la autonomía y la gestión escolar. Asimismo, en la dirección residen otras responsabilidades relacionadas, como guiar, orientar, dirigir y 
organizar a integrantes de las comunidades escolares para asumir diversas tareas que posibilitaran que las escuelas pudieran alcanzar los propósitos definidos en la Ruta de Mejora. En tanto instrumento de Diagnóstico, Planeación, Monitoreo y Evaluación, la comunidad escolar debía tomar decisiones para llevar a cabo la Ruta de Mejora de acuerdo con su contexto y condiciones para impulsar el aprendizaje, disminuir la deserción y abandono escolar. De modo tal que la construcción de esa Ruta permitiese a largo plazo, contribuir a la mejora de la calidad en la educación básica en México.

Sirva este escenario para presentar, en el siguiente apartado, tres de los programas educativos de carácter estratégico que la administración federal anterior emprendió durante su gestión. Cabe decir que la selección del Programa de la Reforma Educativa, el Programa Escuelas de Tiempo Completo y el Programa Nacional de Convivencia Escolar obedecen a que, de manera puntual, cada uno de ellos expresa la singular visión instrumental y pragmática de la participación social que caracterizó la reforma educativa 2012-2018.

\subsection{La participación social en los programas estratégicos de la reforma educativa}

Una de las posibles vías para conocer la noción de participación social impulsada por la reforma educativa del 2012-2018 es el análisis de los programas estratégicos que la tuvieron como uno de sus componentes o ejes principales. Entre esos programas se encuentra el Programa de la Reforma Educativa (PRE), que fue creado en 2014 bajo la denominación de Programa Escuelas de Excelencia para Abatir el Rezago Educativo. En el ciclo escolar 2015-2016 fue renombrado Programa de la Reforma Educativa (PRE) y, en el siguiente ciclo escolar, se fusionó con los programas Escuelas Dignas y Escuelas de Calidad. En el marco de la reforma educativa del 2012-2018, el PRE buscó "atender el problema del deterioro y la inadecuación de las instalaciones en las escuelas de educación básica, acompañado de un insuficiente ejercicio de autonomía de gestión de la comunidad escolar" (El Colegio de México [COLMEX], 2016a, p. 5). De manera puntual, se pretendía que, mediante la transferencia de subsidios federales, fuese empleada por las comunidades de las escuelas públicas de educación básica para emprender, mediante el fortalecimiento de su autonomía, el desarrollo de la Ruta de Mejora en lo concerniente al mejoramiento de la infraestructura y del equipamiento de los centros educativos. La participación de la comunidad escolar fue un elemento esencial para el funcionamiento del Programa que, aunque se reducía al cumplimiento de diversas actividades concretas por parte de las 
familias, también convocaba la colaboración del Consejo Escolar de Participación Social, bajo la supervisión de la autoridad escolar. Todos los actores involucrados debían estar en comunicación para planear en qué y cómo se habrían de utilizar los recursos, así como para plantear la forma en que debían avalar la solicitud formal y asumir la responsabilidad de rendir cuentas de su uso para atender las condiciones físicas y de equipamiento (componente 1), y del subsidio, para apoyar la autonomía de gestión escolar (componente 2).

Este programa fue sometido a varios diagnósticos y evaluaciones durante su implementación, como se constata en el Diagnóstico Ampliado 2015 del Programa de la Reforma Educativa (Gobierno de los Estados Unidos Mexicanos, Secretaría de Educación Pública, 2015), la Evaluación de Diseño Programa de la Reforma Educativa 2016 (COLMEX, 2016), la Evaluación de Consistencia y Resultados 2017 Programa de la Reforma Educativa (Investigación en Salud y Demografía [INSAD], 2017), la Ficha de Monitoreo 2015--2016 (CONEVAL, 2016) y, por último, la Ficha de Monitoreo y Evaluación 2017-2018 (CONEVAL, 2018), entre otros. Pero, a pesar de la relevancia de la participación social en el PRE, este componente no siempre se sometió a la evaluación, como ejemplifican las Fichas de Monitoreo y Evaluación aplicadas por el Consejo Nacional de Evaluación de la Política Social. Paradójicamente, la retórica que suele acompañar la participación social en las acciones gubernamentales se expresó aquí cuando se señaló repetidamente, la importancia de fomentar una participación social de calidad, concebida como aquella que posibilitaba la consecución de los objetivos vinculados con la mejora y la equidad en las escuelas. En los lineamientos del Programa de la Reforma Educativa se materializaba esa intención con la definición puntual de las funciones, responsabilidades y expectativas sobre la participación de los diferentes miembros de las comunidades, ya fuese a nivel individual o colectivo, para garantizar los resultados esperados.

Cabe añadir que, en las evaluaciones citadas arriba, se subrayó la necesidad de invertir en la capacitación de la comunidad escolar para que su intervención tuviese mejores frutos. Si no se contaba con esa capacitación, se argumentaba que se podría producir y reproducir una autonomía orientada por uno o varios agentes externos, así como una autonomía sobreregulada y acotada por los lineamientos operativos del programa que podrían derivar en la configuración de una autonomía de la gestión escolar simulada. A esa observación se sumaron otros problemas que suelen estar presentes cuando el objeto a examinar son procesos de participación social de diversos actores que, de forma individual o 
colectiva, intervienen en la implementación de un programa (Giro, 2010). En el caso del PRE, los desafíos en torno a la necesidad de indicadores específicos, que diesen cuenta, por un lado de la capacidad, la calidad y la efectividad de las decisiones tomadas; y, por otro, de las acciones emprendidas en la implementación del programa, por parte de la gama de actores, quedaron únicamente mencionados y nunca fueron considerados.

Ahora bien, el Programa Escuelas de Tiempo Completo (PETC) aunque surgió como un proyecto piloto en el año 2007, ha sido un programa que ha sido reformulado en diferentes administraciones federales. De acuerdo con el espíritu de la reforma educativa del 2012-2018, este Programa se reimpulsó con la intención de proporcionar mayores oportunidades de aprendizaje y de desarrollo integral mediante la ampliación y uso eficaz de la jornada escolar para la realización de actividades académicas, culturales y deportivas. El supuesto es que dicha reorganización de la jornada escolar contribuiría a la disminución del abandono escolar y favorecería la retención del alumnado de la educación básica. Uno de los rasgos distintivos que se añadieron a este programa fue el conjunto de acciones relativas al suministro de alimentos en la jornada escolar con el fin de promover el cuidado de la salud y el desarrollo de hábitos alimenticios adecuados. Por la reforma educativa, la obligación de las autoridades educativas, por garantizar el incremento paulatino del padrón de escuelas de este programa, quedó establecida en la Constitución Política de los Estados Unidos Mexicanos (Gobierno de los Estados Unidos Mexicanos, 2013. En los lineamientos operativos del programa, correspondientes al último ciclo escolar de la administración anterior 2017-2018, se reiteró la importancia de fomentar la calidad educativa con un enfoque de equidad para asegurar el avance permanente de los aprendizajes, mejorar las prácticas de enseñanza, impulsar el trabajo colaborativo y colegiado, fortalecer la autonomía de gestión escolar, entre otros propósitos.

En este programa, el monto que se asignaba a cada escuela se utilizaba para pagar el apoyo al personal docente y directivo que laboraban de manera extra a su jornada laboral. Los otros rubros, que también recibían un porcentaje importante de los recursos federales, eran para mejorar las condiciones físicas de los espacios educativos y para el pago de asesorías y de capacitación. Los recursos también se podían emplear para fortalecer la autonomía de gestión por medio del acondicionamiento y equipamiento del inmobiliario e infraestructura; la adquisición de materiales educativos; la puesta en marcha de acciones para evitar el abandono y rezago, así como para incrementar el aprovechamiento escolar; el servicio de alimentos; e, incluso, para la implementación, supervisión y evaluación del mismo 
programa. Cabe subrayar que este programa tenía diferentes componentes que no se podían llevar a cabo si no se activaban diversos procesos de participación social concretos, entre ellos se consideraban la organización y el funcionamiento de los comedores escolares; el seguimiento y monitoreo de las acciones realizadas a través de la contraloría social y la rendición de cuentas, y el involucramiento de madres y padres de familia en la educación de sus hijos.

De manera más acentuada que en los diagnósticos y evaluaciones del Programa de la Reforma Educativa, en los estudios del PETC, que fueron realizados, en su gran mayoría, por el Consejo Nacional de Evaluación de la Política de Desarrollo Social (CONEVAL), la participación social fue concebida como un tema secundario. El único análisis que se enfocó en los procesos implementados por el PETC, que incorporó la participación, estuvo a cargo de la Secretaría de Educación Pública, y fue publicado en febrero de 2017 (Gobierno de los Estados Unidos Mexicanos, SEP, 2017b). Dicho estudio se hizo a través de la aplicación de un cuestionario al grupo responsable de la coordinación estatal del programa y una encuesta nacional aplicada a integrantes del equipo directivo $(20,449)$, docente $(18,434)$, madres y padres de familia $(19,292)$ para conocer cuestiones asociadas a la planeación, las solicitudes de apoyos, la selección de beneficiarios, la entrega de apoyos, la producción de bienes y servicios, la contraloría y satisfacción de población usuaria, así como el seguimiento, la evaluación y el monitoreo. Los resultados reiteraron que este programa sí contribuía de manera importante al mejoramiento de los aprendizajes, disminución de la reprobación y rezago escolar. No obstante, se identificó como necesario fortalecer procesos relacionados con el conocimiento de la normatividad y los lineamientos del programa, la distribución y entrega de recursos, la capacitación permanente para difundir y comunicar los resultados alcanzados, el aseguramiento de los servicios de alimentación y la disponibilidad de comedores para población en condición de vulnerabilidad, entre otros.

El tercer programa es el Programa Nacional de Convivencia Escolar (PNCE) surgió a raíz de la lamentable muerte en mayo de 2014 de un alumno de secundaria técnica pública, localizada en el estado de Tamaulipas al norte del país. En respuesta a ese suceso, las autoridades educativas de los 31 estados, la Administración Federal de los Servicios Educativos en el Distrito Federal y la Secretaría de Educación Pública firmaron días después un convenio de coordinación para emprender quince acciones con el fin de combatir la violencia en las escuelas de educación básica en México. Dentro del conjunto de acciones dirigidas a la prevención de la violencia escolar, que conformaron la Estrategia Nacional de 
Combate a la Violencia Escolar, se puede advertir la diversidad de objetivos que acompañarían esos esfuerzos por parte de las autoridades educativas federales, estatales y locales del país, así como también entre diferentes actores gubernamentales y no gubernamentales interesados en la educación.

A esos hechos se remontan los orígenes del PNCE, que operó en calidad de Proyecto Piloto A favor de la Convivencia Escolar entre ciertas escuelas de educación básica desde el ciclo escolar 2014-2015. Fue el ciclo escolar 2016-2017 cuando nació el Programa Nacional de Convivencia Escolar en el marco de la Política Nacional para una Escuela Libre de Acoso Escolar. Este Programa tuvo como objetivo general impulsar la creación de ambientes de convivencia escolar armónica, pacífica e inclusiva para prevenir el acoso escolar y contribuir a la calidad de los aprendizajes en la educación básica y la formación integral de todos los grupos de la población. El Programa tuvo cobertura nacional en las escuelas públicas de nivel básico. En cuanto a sus componentes, se consideraron apoyos técnicos y financieros. El primer tipo de apoyo era otorgado por la Secretaría de Educación Pública a las autoridades estatales locales en cuatro cuestiones: a) el fortalecimiento de las competencias sobre la convivencia escolar, el desarrollo de habilidades socioemocionales necesarias para la participación de los miembros de la comunidad escolar que contribuyesen, a su vez, a la promoción de la permanencia, inclusión y mejora en el logro escolar; b) la integración de acciones para el fortalecimiento de la mejora de las competencias de lectura, escritura y matemáticas; la normalidad mínima de operación escolar; disminución del rezago y abandono escolar; c) la aplicación de protocolos para la prevención, detección y actuación en casos de abuso sexual infantil, acoso escolar y maltrato en las escuelas de educación básica; entre otros. En el diseño del PNCE, la participación social, y particularmente los CEPS, no parecen tener un papel importante en el éxito de la intervención. En contraste, la convocatoria a la participación de madres y padres de familia, y del alumnado, se hace por las decisiones, necesidades y acciones previstas por otros actores.

Este Programa fue sometido a diferentes evaluaciones, tales como la Evaluación de Consistencia y Resultados PNCE 2017-2018 (COLMEX, 2017); el Diagnóstico Ampliado 2015 del PNCE (Gobierno de los Estados Unidos Mexicanos, SEP, 2016); la Evaluación de Diseño PNCE (COLMEX, 2016b). De nueva cuenta, la participación social no ocupó ningún lugar relevante en dichas evaluaciones. En contraste, entre los rubros que fueron evaluados se encuentran los materiales distribuidos y los talleres impartidos entre diferentes integrantes de las escuelas adscritas al programa. Con todo, se emitió la recomendación de que era 
necesario que, en el diagnóstico, se justificase con mayor precisión el tipo de intervención colectiva que este programa deseaba que surgiera espontáneamente de la convivencia cotidiana.

En síntesis, la implementación de estos tres programas estratégicos se concentró en algún actor o modalidad institucional de participación social: el Programa de la Reforma Educativa se enfocó en los Consejos Escolares de Participación Social; el Programa Escuelas de Tiempo Completo en ciertos comités dentro de estos Consejos; y, por su parte, el Programa Nacional de Convivencia Escolar en madres y padres de familia. En general, las tareas y responsabilidades fueron otorgadas en cuestiones tales como la autonomía de la gestión, la contraloría, el seguimiento a la normalidad mínima y otras condiciones favorables al funcionamiento educativo, así como el impulso de la cultura de la transparencia y rendición de cuentas para asegurar el uso correcto de los recursos provenientes de los programas en las escuelas.

\subsection{Argumentos para la discusión de la participación social en la reforma educativa}

La exposición de algunos de los principales rasgos de los programas educativos muestra que la participación social estuvo determinada por la posibilidad de materializar la consecución de los objetivos de la reforma educativa emprendida entre 2012-2018 en México. La visión instrumental y pragmática de la participación fue encarnada a través de las tareas que fueron asignadas a ciertos integrantes de las comunidades escolares y determinadas modalidades institucionales. Esta noción se agudizó por el modelo de autonomía de la gestión escolar de dicha reforma, ya que enalteció el liderazgo directivo en materias como la planeación, organización y uso de los recursos técnicos, financieros y materiales provenientes de programas estratégicos, como el Programa de la Reforma Educativa, el Programa Escuelas de Tiempo Completo y el Programa Nacional de Convivencia Escolar.

Esta perspectiva contrasta con otras experiencias recientes que también han buscado la mejora educativa, pero mediante la gestión pedagógica y participativa, a la par de la democratización de la escuela y el fortalecimiento de relaciones intensas entre la familia y la comunidad (Bolívar, 2018). En ellas, se suele asumir que la colaboración es un dispositivo para aprender y solucionar problemas, fomentar la cooperación y constituirse como una vía estratégica para el desarrollo personal y profesional de todos sus integrantes, donde el 
liderazgo es una práctica amplia, extensa y distribuida a nivel horizontal entre distintos actores, que no se circunscriben necesariamente al espacio escolar. En contraste, las autoridades educativas mexicanas colocaron el liderazgo directivo por encima de los otros actores individuales y/o colectivos. Enfatizaron que esta figura dispone del conocimiento y experiencia necesarios para conducir la autonomía de la gestión escolar. Con ello, el destacado papel otorgado al cuerpo directivo condujo a reforzar la histórica lógica burocrática y vertical en la gestión escolar que marcó la evolución y consolidación del sistema educativo mexicano.

Al hacer un balance de los desafíos pendientes en materia de participación social es pertinente enfocarse en los tipos de actores individuales y en algunas modalidades institucionales que intervienen. El Estado mexicano, si bien, buscó recuperar la rectoría en materia educativa, fundamentalmente, a través de las nuevas reglas respecto a la evaluación educativa y al servicio profesional docente, también buscó garantizar el derecho a la educación con calidad y equidad. Sin embargo, esto no podía materializarse al marginar al estudiantado y convertirlo en un mero observador pasivo de las profundas transformaciones de las escuelas y del sistema educativo. Con ello, se le restó a las escuelas públicas mexicanas la posibilidad de convertirse en el espacio privilegiado para el ejercicio cotidiano de la educación para la vida democrática. Según los representantes de las Voces del Alumnado, la puesta en práctica de esa educación en las escuelas es crucial en el desarrollo individual; por ello, no debe esperar hasta que las NNyA tengan la edad estipulada en la Constitución Política para ejercer sus derechos como ciudadanos (Fielding, 2007, 2011; Lundy, 2007; MackBeath, 2006; Mitra y Serriere, 2012; Mockler y Groundwater-Smith, 2015; Noyes, 2005; Tisdall, Kay y Davis, 2004). Así, a pesar de los importantes cambios en los estilos de educación y de crianza de NNyA, de la movilización de una amplia gama de actores de distinta escala a favor de la protección y garantía de sus derechos, plasmada en diversos marcos legislativos y políticas públicas propagadas desde las últimas décadas, el ejercicio del derecho a la participación de NNyA en las escuelas mexicanas es todavía una promesa incumplida.

Acerca de padres y madres de familia, destaca su invocación permanente en diversos programas estratégicos que comenzaron a desplegarse en los primeros años de la administración encabezada por Enrique Peña Nieto, y que terminaría siendo más visible con las formas en que se les convocó para participar en el Proyecto de la Escuela al Centro y el Nuevo Modelo Educativo de la Educación Obligatoria. Por ejemplo, en este modelo se partió 
del supuesto de que el mejoramiento de la educación demandaba la participación de todas las personas, tal como se estableció en tres de los cinco ejes que lo conformaron, a saber: el modelo de la escuela al centro del sistema educativo, la inclusión y la equidad, así como la gobernanza del sistema educativo. No obstante, a diferencia de lo que ocurrió con el alumnado, en el caso de madres y padres de familias, se pretendió eliminar las barreras que impedían su participación en las escuelas, sobre todo aquella participación que la reforma educativa exigía para la consecución de los objetivos establecidos vinculados con la autonomía de la gestión, la rendición y transparencia de cuentas y el aseguramiento de la normalidad del servicio educativo.

Respecto a las modalidades institucionales de participación social, los Consejos de Participación Social, en especial los del nivel escolar, fueron objeto de mayor atención por parte de las autoridades que impulsaron la reforma educativa entre 2012 y 2018 . En cuanto a las Asociaciones de Padres de Familia, se puede decir que, aunque su normatividad no fue modificada, en diversos documentos operativos de la política educativa se les invitaba para asumir la realización de tareas relacionadas con la implementación de los programas educativos. Sin embargo, en el caso de las Sociedades de Alumnos, ni su marco normativo fue modificado ni tampoco fueron convocadas prácticamente en nada ni en ningún tópico de la reforma. Por otro lado, los Consejos Técnico Escolares, desde la administración previa a la que impulsó la reforma educativa, habían sido objeto de un replanteamiento especialmente operativo más que normativo, que se orientó a la organización de sus actividades a lo largo del ciclo escolar. A raíz de la reforma, este replanteamiento tuvo como referencia principal la construcción e implementación de la Ruta de Mejora. En este contexto, es indiscutible la enorme atención que se concentró en los Consejos de Participación Social, en tanto figura emblemática de la participación social desde principios de los años noventa (Zurita, 2015, $2018 b ; 2018 c$ ). No obstante, el Acuerdo Secretarial 716 y versiones posteriores (Gobierno de los Estados Unidos Mexicanos, Secretaría de Educación Pública, 2014a; 2016, 2017a), expresaron cómo el gobierno federal asumió el reto para impulsar su simplificación y actuación que, como se dijo antes, son aspectos que habían sido, desde su nacimiento, el blanco de múltiples críticas. A pesar de esto, no se solucionaron aquellas otras cuestiones que han impedido que operen adecuadamente, tales como el diseño inadecuado para la conformación y operación de Consejos a nivel Municipal y Estatal, los escasos recursos para funcionar, el desinterés en la apertura de los Consejos Escolares a la participación del alumnado, ya sea individual o colectivo, entre otras (Zurita, 2015, 2018c). Al final, a pesar de 
los cambios incorporados en los diferentes Acuerdos Secretariales que buscaron promover la simplificación y actuación de los Consejos, en los lineamientos de los programas educativos, no se logró materializar esos propósitos dado que su implementación se construyó sobre el abigarrado y complejo diseño de su constitución, organización y funcionamiento.

En adición a ello, desde que los Consejos de Participación Social fueron creados por el Acuerdo Nacional de Modernización de la Educación Básica y Normal en 1992, los esfuerzos gubernamentales se han concentrado en su operación a nivel escolar, por lo cual su seguimiento y monitoreo han descansado en registrar su instalación y sobrevivencia a lo largo del ciclo escolar. Este hecho ejemplifica lo que la literatura abocada al análisis de los Consejos ha identificado en nuestras sociedades respecto a la consideración de indicadores en los cuales predominan los criterios de funcionalidad sobre otros abocados a su calidad y capacidad técnica para intervenir eficaz y eficientemente en procesos de toma de decisión relevantes, así como en su intervención en el fortalecimiento de la participación ciudadana (Cunill, 2012; Medina-Guce, 2018). Este déficit es todavía más agudo porque está lejana la incorporación de enfoque del derecho a la participación en la evaluación de los procesos participativos en las escuelas. Sin ese enfoque es imposible disponer de un referente para conocer cuánto y qué más se necesita para hacer realidad ese derecho de manera indistinta entre todos los individuos y poblaciones sin ningún tipo de excepción, exclusión, discriminación ni desigualdad (UNESCO, 2017).

Ahora bien, desde la perspectiva de este ensayo, el impulso de la participación social de la administración federal anterior deja algunas lecciones para futuros gobiernos y autoridades educativas que ahora conviene puntualizar. En lo concerniente a las modalidades de participación social, una de las principales conclusiones es que continúan sin configurarse como verdaderos espacios para la autonomía de las diferentes iniciativas participativas (Cunill, 2012). La experiencia de la reforma educativa mexicana del 2012-2018 expresa a cabalidad cómo el Estado continuó determinando quiénes podían participar, a quién representaban, cómo, cuándo y para qué participarían. Con ello, de nuevo se le negó a los Consejos de Participación Social cualquier oportunidad para responder de forma directa y efectiva las demandas sociales en el espacio escolar. De manera que continuaron estando expuestos a la cooptación por parte de los intereses arraigados en el sistema educativo y en el gobierno e, incluso, expuestos a emprender experiencias sociales que eventualmente reprodujeran pautas de exclusión social en los ámbitos locales. Así, aun cuando la participación de múltiples actores fue convocada para asumir tareas relacionadas con la 
contraloría social, la transparencia y la rendición de cuentas, en particular los Consejos de Participación Social, no lograron expandir el espacio para el aseguramiento de la autonomía de la sociedad organizada frente al Estado, ni tampoco consiguieron fomentar la deliberación colectiva ni la capacidad de toma de decisiones relevantes para el ámbito escolar y para el sistema educativo (Cunill, 2012).

Una segunda conclusión es que, dada la complejidad de la participación social en la educación y en las escuelas, no resulta adecuado analizarla con modelos que hagan alusión a un desarrollo progresivo y secuencial, tal como lo hicieron en su momento autores como Hart (1993), Trilla y Novella (2001) y Font (2004), cuyos modelos inician con un involucramiento sencillo, y que llegan a culminar en procesos participativos más complejos por el tipo de participación y alcance de sus resultados. No obstante, las investigaciones recientes han revelado que estudiar la participación social desde esa perspectiva, no permite advertir la complejidad que le es inherente, más cuando se trata de una institución tan singular como la escolar, donde predominan relaciones verticales, jerárquicas y en permanente tensión por los distintos tipos de responsabilidad, autoridad y poder que poseen sus integrantes, y que además, cuenta con distintos fuentes de legalidad y legitimidad que respalda su ejercicio en la jornada escolar diaria (Zurita, 2015, 2018a, 2018b). En adición a ello, las jornadas escolares reclaman distintos tipos de participación que suelen estar empalmados en el tiempo y espacio, aun cuando pueden ser radicalmente opuestos. Así, la implementación de un programa puede traducirse en la exigencia de un gran involucramiento y responsabilidad de madres y padres de familia respecto al mantenimiento y mejora de la infraestructura escolar, el control de los recursos financieros recibidos por parte de las autoridades educativas (como se muestra en el Programa de la Reforma Educativa); pero, a la vez, se puede limitar al máximo cualquier intervención de las familias sobre los contenidos de los materiales y talleres de los programas vigentes (como el Programa Nacional de Convivencia Escolar). De igual forma, en ciertos periodos del ciclo escolar, la participación de determinados actores individuales o colectivos es crucial e indispensable, como en la organización de actividades sociales y recreativas; mientras que, en otros momentos del calendario, no se requiere que trabajen con ese propósito. En síntesis, los modelos que conciben la participación como una secuencia ordenada que se despliega en diferentes momentos y que responde a una lógica causal y unidireccional resultan ser poco pertinentes para dar cuenta de las experiencias configuradas en las escuelas (Zurita, 2015, 2018a, 2018b). 
Una tercera conclusión derivada de este ensayo es que la reformulación de la participación social de la reforma educativa del 2012-2018 se sustentó en un discurso donde se adujo que era un medio crucial para mejorar la calidad, los aprendizajes y consolidar el derecho a la educación. No obstante, pronto se advertiría que, por un lado, se impulsaba en las escuelas del tramo obligatorio la participación de determinados actores individuales y colectivos en torno a ciertas acciones para alcanzar determinados objetivos, pero, a la vez, se repudiaba cualquier expresión que se alejase de ella, y más, entre quienes abiertamente se opusieron a la reforma. Con hechos así, se confirmó el carácter polisémico del concepto de la participación social, el cual permite que se haga alusión a ella desde enfoques que fundamentan la formulación de políticas educativas más diferentes y opuestas entre sí.

Como se vio, la administración federal que lanzó, en México, la reforma educativa no fue la excepción (Corvalán y Fernández, 2000; Dagnino, 2006; Font, 2004; López, 2006; Torres, 2001). Así, en diferentes documentos operativos de los programas de la administración federal anterior, se plasmaron varios esfuerzos por precisar la idea de participación social añadiéndole diversos adjetivos. Esto se advierte en distintas menciones en la materia que se encuentran en varios programas estratégicos como la participación social de calidad en el Programa de la Reforma Educativa; o, en el Modelo Educativo para la Educación Obligatoria donde se hablaba de la participación social responsable, la participación social activa y de la participación social corresponsable. Sin embargo, lo que las autoridades educativas consiguieron fue reforzar la retórica sobre la participación social en la educación. A lo largo de su gestión, el gobierno de Enrique Peña Nieto insistió en que la participación social debía ser pensada no como un fin en sí misma, sino como un medio para alcanzar otros objetivos. De manera expresa, quedó indicada esta orientación en las evaluaciones realizadas a los programas estratégicos que acotaron la participación al marco de sus objetivos. Así, la visión pragmática e instrumentalista, incluso, desplazó la idea de la participación social como un fin en sí mismo del sistema educativo, que había circulado en décadas anteriores y que estuvo asociada a la descentralización del sistema educativo, la autonomía escolar, la democratización del espacio escolar y el impulso de la educación para la vida democrática (Barba, 1998; 2007; Latapí, 1998; Zorrilla y Barba, 2008; Zurita, 2013, 2015, 2018a, 2018b, 2018c).

Los altos costos respecto al desarrollo y consolidación de procesos participativos autónomos en la sociedad mexicana son evidentes, pues las escuelas no han logrado ser espacios propicios para el ejercicio de la vida democrática. Sin duda, las consecuencias son 
más graves en el caso de NNyA, ya que a pesar de que existe un marco normativo que reconoce el derecho a la participación en la escuela, la participación sigue siendo asumida como una oportunidad que autoridades escolares le otorgan al estudiantado en ciertos tópicos, generalmente de menor importancia, en periodos breves y siempre bajo el control y la supervisión de personas adultas (Zurita, 2013, 2018b, 2018c). Para la reforma educativa que aquí interesa, conviene decir que las Sociedades de Alumnos continuaron siendo ignoradas socialmente en el Proyecto de la Escuela al Centro y del nuevo Modelo Educativo para la Educación Obligatoria. En este sentido, si bien, la reforma fomentó el derecho a la educación con calidad y equidad, no fue incluyente desde la perspectiva de la participación de NNyA. Según la UNESCO (2017), las dificultades que enfrenta el alumnado para participar en las escuelas nacen de las propias maneras en que está organizado el sistema educativo, de las formas en que se enseña y se busca que aprendan, de los ambientes escolares, así como de las modalidades en que se apoyan, estimulan y evalúan los avances de los estudiantes. Desde esta perspectiva, la reforma educativa acentuó la deuda histórica que el sistema educativo mexicano tiene con el estudiantado.

En cuanto a la participación de madres y padres de familia, promovida por la reforma, se convirtió en una renovada expresión subordinada y circunscrita a la operación de programas. Madres y padres de familia fueron convocados a participar de formas determinadas y en tópicos específicos de la gestión escolar que fueron decididos por las autoridades educativas y escolares. De este modo, la participación se supeditó a los objetivos donde incidían en cuestiones que antes les fue vetado, como asegurar la normalidad del servicio educativo para contrarrestar el poder y la autoridad que pudiese tener la planta docente y directiva de las escuelas. Cabe agregar que, aunque las Asociaciones de Padres de Familia también fueron invitadas para llevar a cabo tareas, fueron notoriamente más visibles los permanentes llamados a madres y padres de familia para asumir un papel protagónico en la reforma educativa.

Finalmente, los Consejos de Participación Social vivieron una regulación normativa, durante unos cuantos años, que nunca habían experimentado en su historia. La publicación y modificación, en varias ocasiones en el sexenio de los Acuerdos Secretariales que buscaron regular su constitución, organización y funcionamiento, muy pronto revelaron ser esfuerzos orientados a la consecución de los propósitos de la reforma en menoscabo de una anhelada conformación como espacios para la participación democrática en las escuelas y en el sistema educativo mexicano. Detrás de cambios importantes, los Consejos continuaron 
teniendo un diseño complejo y abigarrado en su conformación, funcionamiento y, sin duda, en su regulación. Además, a la vez que se incrementaron las tareas y funciones de los Consejos, especialmente de los Consejos Escolares, se restringieron todavía más sus capacidades autónomas de decisión y actuación. En general, la participación social, que fue concebida de una manera pragmática e instrumental por los promotores de la reforma educativa del 2012-2018, se convirtió en un mecanismo crucial para legitimar las decisiones y acciones del Estado mexicano.

\section{Reflexiones finales}

A pocos meses de su comienzo, la administración de Enrique Peña Nieto impulsó un conjunto de profundos cambios en la historia del sistema educativo nacional con el fin de que el Estado mexicano recuperase la rectoría de la educación a través del establecimiento de nuevas reglas en torno a la evaluación educativa y el servicio profesional docente. En las secciones previas de este ensayo se mostró que, en la administración del gobierno federal 2012-2018, se extendió una visión singular de la participación social en el marco de la reforma educativa. Por ahora, conviene concluir señalando que, a pesar de la congruencia existente acerca de la idea de participación social en la reforma educativa que aquí fue analizada tomando como referencia tres programas educativos, hubo otros desafíos que se convirtieron en déficits de la reforma. Entre ellos sobresale el hecho de que, a pesar de los esfuerzos emprendidos por instaurar la evaluación educativa en el tramo obligatorio, se manifestó la incapacidad de las autoridades e instancias gubernamentales por asegurar que el componente de participación social se sometiese al análisis riguroso del diseño, implementación y resultados alcanzados en los programas federales, y por garantizar el uso de indicadores que diesen cuenta de la calidad, capacidad y efectividad de los procesos participativos desplegados, en lugar de continuar contabilizando el número de Consejos instalados o el número de las sesiones realizadas durante el ciclo escolar.

Como en este ensayo se argumenta, la administración mexicana del sexenio anterior difundió una visión pragmática e instrumental de la participación social en las escuelas del tramo obligatorio, que la incorporó como un referente legitimador, limitada a cumplir un papel accesorio en la formulación y operación de políticas y programas e, incluso, fue concebida de nueva cuenta, como un mero componente administrativo y burocrático en el sistema educativo nacional. Sin embargo, los costos más altos que generó esta reforma, en materia de participación social, tienen que ver, sobre todo, con la preminencia del Estado en la 
generación de estas iniciativas, la producción y reproducción de una seudo autonomía social como también de una participación subordinada, simulada y pasiva que, en el caso de ciertos miembros de las comunidades escolares, como el alumnado, se convierte en el desconocimiento y rechazo a la titularidad y ejercicio de su derecho a la participación en las escuelas.

Esa visión no se desmanteló del todo con la llegada del actual gobierno federal, a cargo de Andrés Manuel López Obrador en el año 2018. Una de las primeras acciones de este presidente de la República, anunciada, inclusive desde su campaña electoral, fue la abolición de la reforma educativa de Peña Nieto, especialmente en lo concerniente a la evaluación educativa y al servicio profesional docente. A pesar de llevar a cabo esas iniciativas, en la administración de López Obrador, continuó la implementación de varios programas estratégicos, como el Programa Nacional de Convivencia Escolar y la desaparición de otros, como el Programa de la Reforma Educativa. No obstante, sí introdujo algunos cambios en torno a los Consejos de Participación Social, tales como: la creación de los Comités Escolares de Administración Participativa, cuyo principal cometido es la dignificación de los planteles educativos y la paulatina superación de las desigualdades entre las escuelas del país; la reducción del tipo y número de integrantes y de las funciones de los diferentes Consejos; la eliminación de la obligatoriedad establecida sexenio tras sexenio desde principios de la década de los años noventa respecto a la instalación de los Consejos a nivel escolar, municipal, estatal y nacional, entre otros. Si bien, estos cambios no son menores, por ahora no se advierten esfuerzos serios por impulsar una participación autónoma e independiente de actores individuales o colectivos en las escuelas mexicanas, con los costos negativos que esto implica para el reconocimiento y ejercicio del derecho a la participación en el sistema educativo mexicano. Es más, aunque se ha señalado que un objetivo prioritario en materia educativa es fortalecer la rectoría del Estado y la participación de todos los sectores y grupos de la sociedad, las acciones emprendidas después de un par de años muestran que lo que se busca consolidar es la rectoría del gobierno federal en menoscabo de experiencias y procesos participativos espontáneos y democráticos. Sin embargo, el análisis puntual de esta experiencia será objeto de futuros trabajos. 


\section{Referencias}

Barba, Bonifacio. (1998). La formación de valores y la participación social. En: Pablo Latapí Sarre (Coord.), Un siglo de educación en México (pp. 240-281; Tomo I). México: Fondo de Cultura Económica.

Barba, Bonifacio. (2007). Las (im)posibilidades de la formación ciudadana en México. REICE. Revista Iberoamericana sobre Calidad, Eficacia y Cambio en Educación, 5(4), 51-69. Recuperado de http://rinace.net/arts/vol5num4/art3.pdf

Bolívar, Antonio. (2018). Nueva gobernación en educación y dinámicas para la mejoría: presión versus compromiso. En María de Lourdes, Pinto de Almeida, Marilda Pasqual Schneider y Jaime Morales Vázquez (Comps.), Estado, políticas públicas y educación (pp. 23-56), Ciudad Autónoma de Buenos Aires; CLACSO. Recuperado de http://biblioteca.clacso.edu.ar/clacso/se/20180323034026/Estado politicas publicas e ducacion.pdf

Consejo Nacional de Evaluación de la Política Social. (CONEVAL). (2016). Ficha de Monitoreo 2015-2016. Programa de la Reforma Educativa. México, CONEVAL SEP. Recuperado de https://www.gob.mx/cms/uploads/attachment/file/152737/U082 Ficha de Monitoreo y Evaluacio n 2015-2016.pdf

Consejo Nacional de Evaluación de la Política Social. (CONEVAL). (2018). Ficha de Monitoreo 2017-2018 Programa de la Reforma Educativa. México: CONEVAL SEP. Recuperado de https://www.gob.mx/cms/uploads/attachment/file/390869/U082 Ficha de monitoreo y evaluacio n 2017 - 2018.pdf

Corvalán, Javier. y Fernández, Gabriela. (2000). Apuntes para el análisis de la participación en experiencias educativas y sociales. Revista Latinoamericana de Estudios Educativos (México), 30(4), 9-50. Recuperado de http://www.redalyc.org/pdf/270/27030402.pdf

Cunill Grau, Nuria. (2012). ¿Qué ha pasado con lo público en los últimos 30 años? Revista del CLAD Reforma y Democracia, (52), 1-23. Recuperado de http://old.clad.org/portal/publicaciones-del-clad/revista-clad-reformademocracia/articulos/052-febrero-2012/Cunill.pdf

Dagnino, Evelina. (2006). Sociedad civil, participación y ciudadanía: ¿de qué estamos hablando? En Ernesto Isunza Vera y Alberto Olvera (Coords.), Democratización, rendición de cuentas y sociedad civil: participación ciudadana y control social (pp. 223242). México: H. Cámara de Diputados LIX Legislatura / CIESAS / Universidad Veracruzana / Miguel Ángel Porrúa.

Doepke, Matthias. y Zilibotti, Fabrizio. (2019). Love, Money, and Parenting: How Economics Explains the Way We Raise Our Kids. USA: Princeton University Press. 
El Colegio de México (COLMEX.). (2016a). Evaluación de Diseño Programa de la Reforma Educativa. México: SEP y El Colegio de México. Recuperado de https://www.gob.mx/cms/uploads/attachment/file/131999/U082 MOCyR Informe Final. pdf

El Colegio de México. (COLMEX). (2016b). Evaluación de Diseño PNCE. México. Recuperado de https://www.gob.mx/cms/uploads/attachment/file/131987/S271 MOCyR Informe Final. $\underline{\text { pdf }}$

El Colegio de México. (COLMEX). (2017). Evaluación de Consistencia y Resultados PNCE 2017-2018. México. Recuperado de https://www.gob.mx/cms/uploads/attachment/file/344595/Informe Final S271 Programa Nacional de Convivencia Escolar.pdf

Elizondo Mayer-Serra, Carlos. (2017). Reforma de la Constitución: la economía política del Pacto por México. Revista Mexicana de Ciencias Políticas y Sociales, 62(230), 21-50. Recuperado http://www.revistas.unam.mx/index.php/rmcpys/article/view/58765/52400

Epstein, Joyce, Clark, Laurel, Salinas, Karen Clark, y Sanders, Mavis. (1997). Scaling up school-family-community conecctions in Baltimore: Effects on student achievement and attendance. Baltimore: CRESPAR / Center on School, Family and Community Partnerships / John Hopkins University.

Fielding, Michael. (2007). Beyond "Voice": New roles, relations, and contexts in researching with young people. Discourse: studies in the cultural politics of education, 28(3), 301310. DOI: https://doi.org/10.1080/01596300701458780

Fielding, Michael. (2011). Patterns of partnership: Student voice, intergenerational learning and democratic fellowship. En Nicole Mockler y Judyth Sachs (Eds.), Rethinking educational practice through reflexive inquiry: Essays in honour of Susan GroundwaterSmith (pp. 61-75). Dordrecht: Springer.

Font, Joan. (2004). Participación ciudadana y decisiones públicas: conceptos, experiencias y metodologías. En Alicia Ziccardi (Coord.), Participación ciudadana y políticas sociales en el ámbito local (pp. 23-42). México, IIS / COMECSO / INDESOL.

Gajardo, Marcela. (1999). Reformas educativas en América Latina. Balance de una década. Chile, PREAL. Recuperado de http://opech.cl/bibliografico/calidad equidad/Reformas educativas balance de decad a PREAL.pdf

Giro, Joaquín. (2010). Construcción de indicadores de participación de los padres en la enseñanza obligatoria. Ponencia presentada en el X Congreso Español de Sociología, organizado por la Federación Española de Sociología. 
Gobierno de los Estados Unidos Mexicanos, Secretaría de Educación Pública (SEP). (1942). Ley Orgánica de la Educación Pública. México. Recuperado de http://www.sep.gob.mx/work/models/sep1/Resource/3f9a47cc-efd9-4724-83e40bb4884af388/ley 23011942.pdf

Gobierno de los Estados Unidos Mexicanos, Secretaría de Educación Pública (SEP). (2013). Lineamientos para la Organización y el Funcionamiento de las Escuelas de Tiempo Completo. México. Recuperado de http://www.sec.gob.mx/coordinacion/uploads/lineamiento\%20petc.pdf

Gobierno de los Estados Unidos Mexicanos, Secretaría de Educación Pública (SEP). (2014a). Acuerdo Secretarial 716 que establece lineamientos para la constitución, organización y funcionamiento de los Consejos de Participación Social en la Educación. $\quad$ Recuperado http://dof.gob.mx/nota detalle.php?codigo=5335232\&fecha=07/03/2014

Gobierno de los Estados Unidos Mexicanos, Secretaría de Educación Pública (SEP). (2014b). Acuerdo Secretarial 717 por el que se establecen los lineamientos para formular los Programas de Gestión Escolar. Recuperado de http://dof.gob.mx/nota detalle.php?codigo=5335233\&fecha=07/03/2014

Gobierno de los Estados Unidos Mexicanos, Secretaría de Educación Pública (SEP). (2015). Diagnóstico Ampliado 2015 del PNCE. México. Recuperado de https://www.gob.mx/cms/uploads/attachment/file/147558/Diagnostico Ampliado.pdf

Gobierno de los Estados Unidos Mexicanos, Secretaría de Educación Pública (SEP). (2016). ACUERDO número 02/05/16 por el que se establecen los Lineamientos para la constitución, organización y funcionamiento de los Consejos de Participación Social en la Educación. Recuperado de http://dof.gob.mx/nota detalle.php?codigo=5436739\&fecha $=11 / 05 / 2016$

Gobierno de los Estados Unidos Mexicanos, Secretaría de Educación Pública (SEP). (2017a). Acuerdo número 08/08/17 que modifica el diverso número 02/05/16 por el que se establecen los Lineamientos para la constitución, organización y funcionamiento de los Consejos de Participación Social en la Educación. Recuperado de https://dof.vlex.com.mx/vid/acuerdo-numero-08-08-692063937

Gobierno de los Estados Unidos Mexicanos, Secretaría de Educación Pública (SEP). (2017b). Análisis de los procesos del Programa Escuelas de Tiempo Completo (PETC). Recuperado https://www.gob.mx/cms/uploads/attachment/file/226696/Ana lisis de los procesos d el PETC Completo-Final.pdf

Gobierno de los Estados Unidos Mexicanos. (2013). Constitución Política de los Estados Unidos Mexicanos. Recuperado de https://drive.google.com/drive/folders/1z1iiVn9rbkiVIZkf5nWkldJfztiplHRC 
Gobierno de los Estados Unidos Mexicanos. (2018). Ley General de Educación. Recuperado de https://www.sep.gob.mx/work/models/sep1/Resource/558c2c24-0b12-4676-ad908ab78086b184/ley general educacion.pdf

Hart, Roger. (1993). La participación de los niños: de la participación simbólica a la participación auténtica. (Ensayos Innocenti № 4). Bogotá: Unicef. Recuperado de https://www.unicef-irc.org/publications/pdf/ie participation spa.pdf

Hornby, Garry y Lafaele, Rayleen. (2011). Barriers to parental involvement in education: an explanatory model. Educational Review, 63(1), 37-52. doi: https://doi.org/10.1080/00131911.2010.488049

Investigación en Salud y Demografía, S.C. (INSAD), (2017). Evaluación de Consistencia y Resultados Programa de la Reforma Educativa. México: SEP. Recuperado de https://www.gob.mx/cms/uploads/attachment/file/365198/Informe Final U082 Programa de la Reforma Educativa.pdf

Latapí Sarre, Pablo. (1998). Un siglo de educación nacional: una sistematización. En Pablo Latapí Sarre (Coord.), Un siglo de educación en México (pp. 21-42). México: Fondo de Cultura Económica. Recuperado de https://sistemaeducativo.files.wordpress.com/2011/02/un-siglo-de-educacion1.pdf

Latapí Sarre, Pablo. (2005). La SEP por dentro. Las políticas de la Secretaría de Educación Pública comentadas por cuatro de sus secretarios (1992 - 2004). México: Fondo de Cultura Económica.

López, María Margarita. (2006). Una revisión a la participación escolar en América Latina, Chile: PREAL. Recuperado de https://bibliotecas.ort.edu.uy/bibid/63101/file/35

Lundy, Laura. (2007). 'Voice' Is Not Enough: Conceptualising Article 12 of the United Nations Convention on the Rights of the Child. British Educational Research Journal, 33(6), 927-942. doi: https://doi.org/10.1080/01411920701657033

MacBeath, John. (2006). Finding a voice, finding self. Educational Review, 58(2), 195-207. doi: https://doi.org/10.1080/00131910600584140

Martínez Bordón, Arcelia. y Navarro Arredondo, Alejandro (Coords.). (2018). La Reforma Educativa a revisión: apuntes y reflexiones para la elaboración de una agenda educativa 2018-2024. Recuperado de http://bibliodigitalibd.senado.gob.mx/bitstream/handle/123456789/4143/Libro reformae ducativa final.pdf?sequence $=1$ \&isAllowed $=y$

McCluskey, Gillean Grassie., Brown, Jane., Munn, Pamela., Lloyd, Gwynedd., Hamilton, Lorna., Sharp, Stephen. and MacLeod, Gale. (2013). Take more time to actually listen': students' reflections on participation and negotiation in school. British Educational Research Journal, 39(2), 287-301. doi: https://doi.org/10.1080/01411926.2012.659720 
Medina-Guce, Czarina. (2018). Substance over Form: Improving Assessments of Local Development Councils (LDCs) and Local Development Investment Programs (LDIPS). Recuperado de https://www.academia.edu/37796471/Policy Note Improving Assessments of Local Development Councils and Local Development Investment Programs

Mitra, Dana L. y Serriere, Stephanie C. (2012). Student Voice in Elementary School Reform: Examining Youth Development in Fifth Graders. American Educational Research Journal, 49(4), 743-774. doi: https://doi.org/10.3102/0002831212443079

Mockler, Nicole. y Groundwater-Smith, Susan (eds.). (2015). Engaging with Student Voice in Research, Education and Community Beyond Legitimation and Guardianship. Cham Heidelberg, New York Dordrecht London, Springer.

Noyes, Andy. (2005). Pupil voice: purpose, power and the possibilities for democratic schooling. British Educational Research Journal, 31(4), 533-540.

Organización de las Naciones Unidas para la Educación, la Ciencia y la Cultura (UNESCO). (2017). Guía para asegurar la inclusión y la equidad en la educación. Francia, Organización de las Naciones Unidas para la Educación, la Ciencia y la Cultura. Recuperado de http://down21chile.cl/cont/cont/2017/336 2 guia para asegurar la inclusion y la equidad en la $\mathrm{e}$ ducacion.pdf

Secretaría de Educación Pública, Subsecretaría de Planeación y Evaluación de Políticas Educativas. Dirección General de Evaluación de Políticas. (SEP, SPEPP, DGEP). (2015). Diagnóstico Ampliado del Programa de la Reforma Educativa. México. SEP. Recuperado https://www.gob.mx/cms/uploads/attachment/file/50174/Diagno stico Ampliado.pdf

Tisdall, E. Kay M. and Davis, John. (2004). Making a Difference? Bringing Children's and Young People's Views into Policy-Making. Children \& Society, 18(2), 131-142.

Torres, Rosa María. (2001). Participación ciudadana y educación. Una mirada amplia y 20 experiencias en América Latina. Quito: Instituto Frónesis. Recuperado de http://edu.jalisco.gob.mx/cepse/sites/edu.jalisco.gob.mx.cepse/files/participacion cdada na unesco.pdf

Trilla, Jaume. y Novella, Ana. (2001). Educación y participación social de la infancia. Revista Iberoamericana de Educación, (36). Recuperado de https://rieoei.org/historico/documentos/rie26a07.htm

Zorrilla, Margarita. y Barba, Bonifacio. (2008). Reforma educativa en México. Descentralización y nuevos actores. Revista Electrónica de Educación Sinéctica, (30), 1-30. Recuperado de https://sinectica.iteso.mx/index.php/SINECTICA/article/view/189

Zurita Rivera, Ursula. (2013). Las oportunidades para participar de las y los adolescentes en las escuelas secundarias públicas en México. Revista Interacções, 9(26), 229-260. Recuperado de https://revistas.rcaap.pt/interaccoes/article/view/3367 
Zurita Rivera, Ursula. (2015). La participación social y los Consejos de Participación Social a partir de la reforma impulsada en el sexenio 2012-2018. En: Marcos Jacobo Estrada (Coord.), Las condiciones de la participación social en la educación en México. Alcances y límites (pp. 93-120). México, El Colegio de Sonora y Juan Pablos Editor.

Zurita Rivera, Ursula. (2018a). Autonomía, Gestión, Participación Social: Desafíos para la Gobernanza Escolar en México. Revista Praxis Sociológica, (23), 255-271. Recuperado de https://dialnet.unirioja.es/servlet/articulo?codigo=6312284

Zurita Rivera, Ursula. (2018b). La participación social en la Reforma Educativa en México: otra oportunidad perdida. En Arcelia Martínez Bordón y Alejandro Navarro Arredondo (Coords.), La Reforma Educativa a revisión: apuntes y reflexiones para la elaboración de una agenda educativa 2018-2024 (pp. 145-167). México: Instituto Belisario Domínguez del Senado de la República. Recuperado de http://bibliodigitalibd.senado.gob.mx/bitstream/handle/123456789/4143/Libro reformae ducativa final.pdf?sequence $=1$ \&isAllowed $=y$

Zurita Rivera, Ursula. (2018c). Desafíos de la participación social en las escuelas y la educación a cien años de la Constitución Política de los Estados Unidos Mexicanos. En: Jesús Adolfo Trujillo Holguín. y Laura Irene Dino Morales (Coords.), Debate legislativo y educación. El artículo tercero a cien años de la Constitución Política de 1917 (pp. 283-300). México, Universidad Autónoma de Chihuahua. Recuperado de https://www.rediech.org/inicio/index.php/publicaciones/otraspublicaciones/descargas/item/1039-debate-legislativo-y-educacio-n-el-arti-culo-terceroa-cien-an-os-de-la-constitucio-n-poli-tica-de-1917/1039-debate-legislativo-y-educacio-nel-arti-culo-tercero-a-cien-an-os-de-la-constitucio-n-poli-tica-de-1917 
Revista indizada en
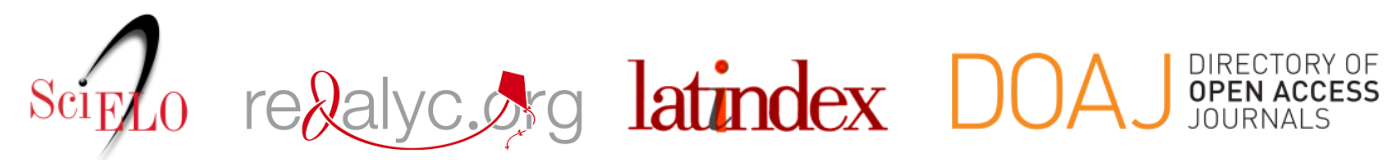

Distribuida en las bases de datos:

- Dialnet

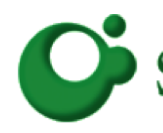
SHERPA/RøMEO

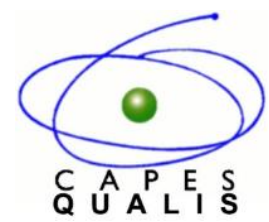

MIAR 